\title{
FLUCTUATIONS SAISONNIERES DU POIDS VIF D'OVINS DJALLONKE TYPE MOSSI DU BURKINA FASO EN RELATION AVEC LE PROFIL FERMENTAIRE DANS LE RUMEN
}

\author{
J. S. ZOUNDI' ${ }^{1}$, L. SAWADOGO ${ }^{2}$, et A. J. NIANOGO3 \\ ${ }^{1}$ Institut de l'Environnement et de Recherches Agricoles (INERA) \\ 04 BP 8645 Ouagadougou 04 BURKINA FASO. \\ e-mail : zoundi@hotmail.com ou zoundi@fasonet.bf \\ 2 UFR - SVT, Université de Ouagadougou, 03 BP 7021 Ouagadougou 03 BURKINA FASO. \\ ${ }^{3}$ Université Polytechnique de Bobo, 01 BP 1091 Bobo-Dioulasso 01 BURKINA FASO.
}

\begin{abstract}
RESUME
Des ovins mâles Djallonké âgés de 10-12 mois utilisant des pâturages naturels, dans le but d'évaluer la contribution des parcours naturels dans l'alimentation des ruminants au sein des systèmes d'exploitation mixte agriculture-élevage, ont été suivis pendant les différentes périodes de l'année dans le plateau central du Burkina Faso. Les niveaux de $\mathrm{N}-\mathrm{NH}_{3}$ enregistrés varient significativement $(\mathrm{p}<0,1 \%)$ avec les périodes de l'année. Les plus fortes valeurs ont été observées en novembre-décembre $(112,8 \mathrm{mg} / \mathrm{l})$. La production totale d'acides gras volatils (AGV) présente également des différences significatives $(p<0,1 \%)$ avec la saison. Les valeurs les plus élevées sont enregistrées pendant la saison de pluies en juin, juillet et août-septembre soit respectivement 24,$25 ; 27,10$ et $26,66 \mathrm{mmol} / \mathrm{l}$. Des variations significatives $(p<0,1 \%)$ sont observées entre les proportions molaires des AGV ; les plus fortes proportions de propionate étant enregistrées en mai-juillet. Les performances pondérales montrent des fluctuations significatives $(p<0,1 \%)$ avec la saison et des corrélations significatives existent entre les GMQ et les proportions molaires de propionate $(r=0,55 ; p<0,1 \%)$ et le rapport acétate/propionate $(r=-0,51 ; p<1 \%)$. II ressort de cette étude que la période post-récolte (novembre-janvier) présente des conditions nutritionnelles favorables pour les animaux. La période pré-récolte (octobre) apparaît très critique $(-22,3 \mathrm{~g} \mathrm{GMQ}$ ) et les stratégies d'alimentation devraient privilégier l'atténuation de cette période de sous-nutrition par des apports complémentaires d'énergie et de protéine, surtout au profit des femelles gestantes ou allaitantes.
\end{abstract}

Mots-clés : ovin Djallonké, acide gras volatil, azote ammoniacal, alimentation naturelle, Burkina Faso

\author{
ABSTRACT \\ SEASONAL FLUCTUATIONS IN LIVE WEIGHT OF BURKINA FASO MOSSI TYPE DJALLONKE SHEEPS \\ IN RELATION TO FERMENTATION PROFILE IN THE RUMEN
}

In order to assess the contribution of natural pasture in ruminants feeding mixed crop-livestock farming systems, male Djallonke sheeps grazing natural pasture were followed through the different periods of the

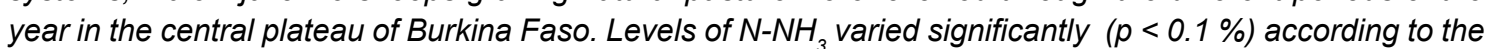
period of the year. The highest values were observed in November and December (112.8 $\mathrm{mg} / \mathrm{l})$. The total production of volatile fatty acids (VFA) also showed significant differences $(p<0.1 \%)$ following the season. The highest values were recorded during the rainy season : 24.25, 27.10 and $26.66 \mathrm{mmol} / \mathrm{l}$, respectively, in june, july, and august-september. Significant variations $(p<0.1 \%)$ were observed with VFA molar proportions. The highest proportions of propionate were recorded from may to july. Live weights showed significant fluctuations $(p<0.1 \%)$ depending on the season, and significant correlations exist between $A D G$ and propionate molar proportions $(r=0.55 ; p<0.1 \%)$ and the ratio of acetate/propionate $(r=-0.51$; $p<1 \%$ ). The study revealed that the post-harvest period (november to january) presented better feeding 
conditions for animals. The pre-harvest period (october) appears to be very critical (-22.3 $\mathrm{g} A D G)$ and animal feeding strategies should tackle that period of malnutrition with nitrogen and energetic supplementation, especially for the benefit of pregnant or lactating females.

Keywords: Djallonke sheep, volatile fatty acid, ammonium nitrogen, natural feeding, Burkina Faso

\section{INTRODUCTION}

Les parcours naturels (pâturages et résidus de cultures) demeurent la principale source d'alimentation des ruminants au sein des systèmes d'exploitation mixte agricultureélevage (Gryseels, 1988). Toutefois, ceux-ci sont reconnus pour leur faible valeur nutritive, plus particulièrement en azote avec des taux constamment en dessous de $1 \%$ en saison sèche (Boudet, 1985 ; Penning De Vries et Djiteye, 1982 ; Savadogo, 2000). Ces genres de substrats, tout en limitant l'ingestion volontaire (Ketelaars et Tolkamp, 1991) offrent des conditions peu propices (notamment la limitation en $\mathrm{N}-\mathrm{NH}_{3}$ ) pour l'activité microbienne dans le rumen (Pritchard et Males, 1982; Grenet et Besle, 1991 ; Komisarczuk et Durand, 1991). Une telle limitation entraîne généralement une faible production d'acides gras volatils, le plus souvent couplée à une forte proportion molaire d'acide acétique, avec comme conséquence, une baisse de l'efficience de l'utilisation de l'énergie par les animaux (Van Houtert, 1993).

Dans le plateau central du Burkina, l'alimentation des ruminants repose essentiellement sur les pâturages naturels, mais ces derniers présentent des insuffisances eu égard aux pertes de poids vif enregistrées en certaines périodes de l'année (Bicaba et al., 1986 ; Zoundi et al., 1994).

Au regard de cette situation nutritionnelle variable selon les saisons de l'année, les animaux ont toujours développé des mécanismes de survie. Ceux-ci sont marqués par de fortes accumulations de tissus (notamment adipeux) en période favorable, leur permettant de minimiser les pertes et de faire face aux besoins vitaux pendant les moments critiques (Zoundi et al., 1994 ; Orskov, 1997).

L'intérêt des pâturages naturels dans les systèmes extensifs d'élevage a été à la base de la conduite de certaines investigations (Guerin et al., 1988). Celles-ci ont permis, d'une part, de connaître la composition des bols alimentaires prélevés sur les pâturages et, d'autre part, de faire un inventaire des principales espèces les plus appétées tout en appréciant les fréquences de pâture. Elles offrent ainsi des indications en matière d'organisation de la gestion des parcours naturels et de certaines espèces végétales en particulier, mais ne permettent pas d'évaluer le statut nutritionnel des animaux en fonction des différentes périodes de l'année.

La présente étude évalue la contribution des parcours naturels en terme de produits de la digestion et leur lien avec les performances pondérales. Elle vise surtout à fournir des indications utiles, nécessaires à l'élaboration de systèmes de complémentation destinés aux animaux utilisant les pâturages naturels comme base de leur alimentation.

\section{MATERIEL ET METHODES}

\section{MILIEU D'ETUDE}

L'étude a été conduite dans une zone du plateau central du Burkina comprise entre les isohyètes 600 et $800 \mathrm{~mm}$. La figure 1 résume la situation pluviométrique du site pendant la période d'étude.

Le système d'exploitation est de type mixte agriculture-élevage et les principales cultures vivrières agricoles sont le mil, le sorgho, généralement cultivés en association avec le niébé.

Les pâturages naturels s'apparentent beaucoup au type sahélo-soudanien décrit par Toutain (1987), avec une dominance de graminées annuelles : Loudetia togoensis (Pilger) C.E. Hubbard, Pennisetum pedicellatum Trin., Andropogon pseudapricus Stapf. La pression agricole sur les terres est très forte et les quelques rares parcours naturels sont constitués essentiellement par les formations suivantes : (a) jachères de courte à moyenne durée avec comme espèces dominantes Pennisetum pedicellatum, Eragrostis tremula 
Hochst., Loudetia togoensis, Piliostigama reticulatum (DC.) Hochst., Guiera senegalensis J.F.Gmel. ; (b) champs de culture avec les résidus de culture (sorgho, mil, niébé, arachide, voandzou, etc...) et quelques adventices comme Alysicarpus ovalifolius (DC.) Guill. \& essentiellement les jachères. En saison sèche (de novembre à mai), les animaux sont laissés en divagation. La pâture a lieu dans les champs de culture (surtout entre novembre et janvier), dans les jachères et les zones inondées. L'abreuvement a lieu directement

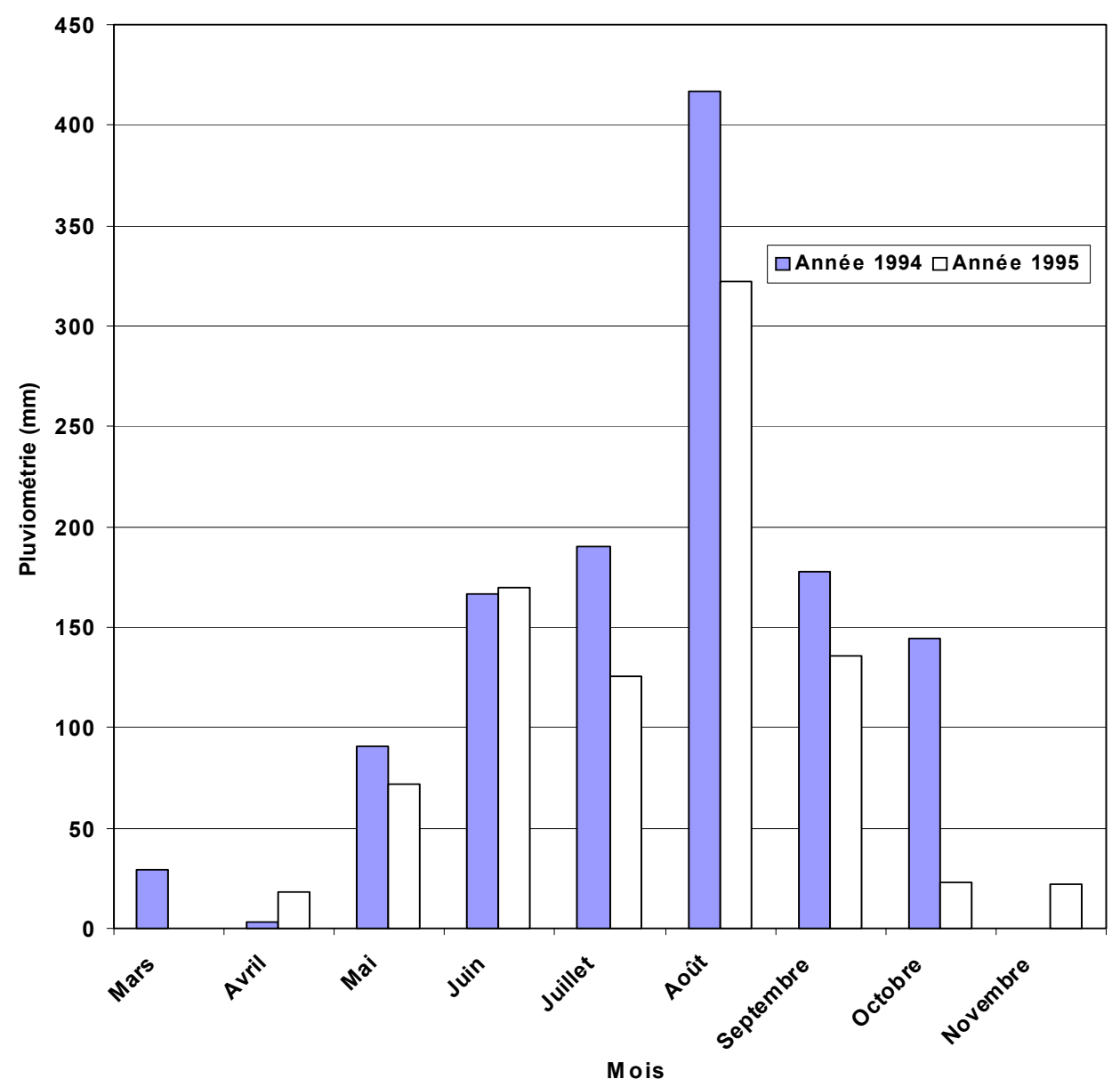

Figure 1 : Pluviométrie enregistrée dans le plateau central Burkinabè pendant la période d'étude

Rainfall recorded in the Burkinabè central plateau during the study

Perr. ; (c) pâturages de zones inondées en bordure des cours d'eau avec Andropogon gayanus Kunth., Andropogon pseudapricus, Vetiveria negritana (Benth.) Stapf., Mitragyna inermis (Willd.) O. Ktze., Anogeisus leiocarpus (DC.) Guill. \& Perr.

\section{DISPOSITIF EXPERIMENTAL}

Les travaux se sont déroulés en milieu réel à partir des troupeaux gérés par les paysans. En saison de pluies, les troupeaux ovins sont gardés et conduits au pâturage. Ils bénéficient d'une pâture journalière d'environ 9 heures $(8 \mathrm{H}$ à $17 \mathrm{H})$. Les pâturages exploités par les animaux pendant cette période sont dans les cours d'eau ou à la ferme pendant les périodes de forte pénurie (de mars à mai). Quelques rares com-pléments, son et sel notamment, sont fournis à certaines catégories : femelles allaitantes, animaux malades ou à l'embouche.

Dix ovins mâles entiers de race Djallonké type Mossi âgés de 10 à 12 mois avec un poids vif moyen initial de 13,92 $\pm 0,73 \mathrm{~kg}$ ont été identifiés à partir d'un troupeau villageois pour les suivis et différentes mesures. Ces animaux sont soumis à la même conduite que le reste du troupeau, et ne bénéficient d'aucune complémentation. 
La pesée des animaux a lieu toutes les deux semaines à $7 \mathrm{H}$ avant le départ pour le pâturage. Les prélèvements de jus de rumen ont également eu lieu une fois toutes les 2 semaines selon les procédures décrites par Preston (1986). Deux mesures sont faites à chaque prélèvement, une le matin à $7 \mathrm{H}$ et l'autre le soir à $17 \mathrm{H}$. Après la mesure du $\mathrm{pH}$, le dosage de l'azote ammoniacal $\left(\mathrm{N}-\mathrm{NH}_{3}\right)$ est fait selon les procédures décrites par Preston (1986). Un échantillon de $20 \mathrm{ml}$ de jus de rumen est ensuite prélevé, acidifié $(\mathrm{pH}=3)$ avec du $\mathrm{HCl}$, et conservé au frais. Le dosage des acides gras volatils (AGV) est fait par chromatographie en phase gazeuse.

Les paramètres considérés pour les traitements des données de l'étude, sont les mois ou les périodes de l'année pour lesquels différents états nutritionnels sont observés. Les variables suivantes ont été mésurées : les poids vifs et les paramètres fermentaires du rumen $(\mathrm{pH}$, azote ammoniacal et acides gras volatils). Chaque animal constitue une répétition, soit un total de 10 répétitions à chaque mesure. La comparaison des moyennes deux à deux en fonction du mois ou des périodes de l'année a été faite selon le test de Student-NewmanKeuls. Les analyses statistiques (analyse de moyenne et corrélation) ont été faites à l'aide du logiciel SAS (1982).

\section{RESULTATS}

\section{NIVEAUX DE pH ET DE N-NH}

Les niveaux d'azote ammoniacal $\left(\mathrm{N}-\mathrm{NH}_{3}\right)$ mesurés (tableau 1) montrent des variations hautement significatives ( $p<0,1 \%$ ) avec les périodes de l'année. L'arrivée de la saison de pluies correspond à une augmentation significative des niveaux de $\mathrm{N}-\mathrm{NH}_{3}$ dans le rumen des animaux, passant ainsi de 30,2 mg/l en moyenne en mars-avril à des taux de $87,7 \mathrm{mg} / \mathrm{l}$ en septembre-octobre. Un deuxième pic apparaît pendant la période novembre-décembre, avec une moyenne de 112,8 mg/l.

Les valeurs de $\mathrm{pH}$ (tableau 1) montrent également des variations hautement significatives ( $p<0,1 \%$ ) avec les périodes de l'année ; les plus fortes valeurs étant enregistrées en saison sèche $(6,64$ et 6,41 respectivement en janvier-février et en mars-avril).

\section{ANALYSE DE LAPRODUCTION D'ACIDES GRAS VOLATILS}

Des variations hautement significatives ( $p<0,1 \%$ ) ont été observées (tableaux 2a et 2b) au niveau de la production totale d'AGV. Les plus fortes valeurs ont été enregistrées en saison de pluies, particulièrement, pendant les mois de juin à août : $17,84 \mathrm{mmol} / \mathrm{l}, 19,76 \mathrm{mmol} / \mathrm{l}$ et $19,34 \mathrm{mmol} / \mathrm{l}$ pour l'acétate, respectivement en juin, juillet et août/ septembre.

L'analyse des proportions molaires des différents AGV montre également des différences hautement significatives avec la période pour l'acétate $(p<1 \%)$, le propionate $(p<0,1 \%)$ et le butyrate $(p<1 \%)$. Les plus fortes proportions de propionate ont été observées respectivement en mai-juillet et en novembre-décembre avec des valeurs atteignant $14,18 \%$ en juin et $14,46 \%$ en Décembre.

Le ratio acétate/propionate (C2/C3) suit la même tendance avec les plus faibles valeurs observées pendant ces deux périodes avec des valeurs de 5,3 en juin et 5,25 en décembre. Ces ratios présentent des variations hautement significatives $(p<0,1 \%)$ avec les périodes de l'année.

Tableau 1 : Gains de poids vif, teneurs en $\mathrm{N}-\mathrm{NH}_{3}$ et ratio acétate/propionate.

Live weight gains, level of $\mathrm{N}-\mathrm{NH}_{3}$ and acetate/propionate ratio.

\begin{tabular}{|c|c|c|c|c|c|c|c|}
\hline \multirow[t]{2}{*}{ Paramètres mesurés } & \multicolumn{6}{|c|}{ Périodes } & \multirow{2}{*}{$\begin{array}{l}\text { Ecart- } \\
\text { Type }\end{array}$} \\
\hline & P1 & $\mathrm{P} 2$ & P3 & P4 & P5 & P6 & \\
\hline G M Q（g) & $17,9 b$ & $28,3 b$ & $43,2 \mathrm{a}$ & $-22,3 c$ & $56,6 \mathrm{a}$ & $47,6 a$ & 12,2 \\
\hline $\mathrm{N}-\mathrm{NH}_{3}(\mathrm{mg} / \mathrm{l})$ & $30,3 \mathrm{e}$ & $58,0 \mathrm{c}$ & $77,4 b$ & $87,7 \mathrm{~b}$ & $112,8 \mathrm{a}$ & $44,2 d$ & 9,4 \\
\hline $\mathrm{pH}$ & $6,41 b$ & $6,17 \mathrm{c}$ & $6,40 \mathrm{~b}$ & $6,34 b$ & $6,35 b$ & $6,64 a$ & 0,08 \\
\hline
\end{tabular}

GMQ : Gain Moyen Quotidien

Les valeurs figurant sur la même ligne et ne portant aucun indice commun sont significativement différentes : $p<0,01 \%$ (pour GMQ) ; $p<0,1 \%$ (pour $\mathrm{N}-\mathrm{NH} 3$ et $\mathrm{pH}$ ), selon le test de Student-Newman-Keuls.

P1 : Mars-Avril ; P2 : Mai-Juin ; P3 : Juillet-Août ; P4 : SeptembreOctobre ; P5 : Novembre-Décembre ; P6 : Janvier-Février.
Means in the same row with different labels are significantly different for the follow levels of significance : $p<0,01 \%$ (for $A D G$ ) ; $p<0,1 \%$ (for $\mathrm{N}-\mathrm{NH} 3$ and $\mathrm{pH}$ ) according to StudentNewman-Keuls Test.

P1 : March-April ; P2 : May-June ; P3 : Jully-August P4: September-October; P5: November-December; $P 6$ : January-February. 
Tableau 2a : Evolution de la production d'acides gras volatils dans le rumen pendant la saison sèche. Pattern of volatil fatty acids production in the rumen during the dry season.

\begin{tabular}{lccccccc}
\hline & \multicolumn{7}{c}{ Mois } \\
\cline { 2 - 7 } & Octobre & Novembre & Décembre & Janvier & Février & Mars & Avril \\
\hline Acétate (mmol/l) & $7,18 \mathrm{c}$ & $16,91 \mathrm{ab}$ & $7,74 \mathrm{c}$ & $16,27 \mathrm{ab}$ & $15,76 \mathrm{ab}$ & $13,00 \mathrm{~b}$ & $15,70 \mathrm{ab}$ \\
Acétate (\%) & $81,02 \mathrm{a}$ & $72,23 \mathrm{~b}$ & $74,69 \mathrm{ab}$ & $73,67 \mathrm{ab}$ & $79,06 \mathrm{ab}$ & $80,97 \mathrm{a}$ & $76,23 \mathrm{ab}$ \\
Propionate (mmol/l) & $0,94 \mathrm{e}$ & $3,08 \mathrm{abc}$ & $1,53 \mathrm{de}$ & $2,41 \mathrm{bcd}$ & $1,64 \mathrm{de}$ & $1,89 \mathrm{cde}$ & $2,05 \mathrm{cde}$ \\
Propionate (\%) & $10,60 \mathrm{abc}$ & $13,10 \mathrm{ab}$ & $14,46 \mathrm{a}$ & $10,90 \mathrm{abc}$ & $8,15 \mathrm{c}$ & $12,23 \mathrm{abc}$ & $10,31 \mathrm{abc}$ \\
Butyrate (mmol/l) & $0,76 \mathrm{~d}$ & $3,45 \mathrm{ab}$ & $1,19 \mathrm{~cd}$ & $3,50 \mathrm{ab}$ & $2,72 \mathrm{abcd}$ & $1,15 \mathrm{~cd}$ & $3,31 \mathrm{ab}$ \\
Butyrate (\%) & $8,38 \mathrm{bc}$ & $14,67 \mathrm{ab}$ & $10,83 \mathrm{abc}$ & $15,43 \mathrm{ab}$ & $12,78 \mathrm{abc}$ & $6,79 \mathrm{c}$ & $13,50 \mathrm{abc}$ \\
AGVTot & $8,88 \mathrm{~d}$ & $23,44 \mathrm{ab}$ & $10,46 \mathrm{~cd}$ & $22,19 \mathrm{ab}$ & $20,12 \mathrm{ab}$ & $16,04 \mathrm{bc}$ & $21,06 \mathrm{ab}$ \\
C2/C3 & $7,76 \mathrm{ab}$ & $5,59 \mathrm{~b}$ & 5,25 & $7,12 \mathrm{~b}$ & $9,82 \mathrm{a}$ & $7,53 \mathrm{ab}$ & $7,69 \mathrm{ab}$ \\
\hline
\end{tabular}

Les valeurs figurant sur la même ligne et ne portant aucun indice commun sont significativement différentes pour : $\mathrm{P}<0,1 \%$ (pour Acétate, Propionate, Propionate (\%), Butyrate, AGVTot, C2/C3); $\mathrm{P}<1 \%$ (pour Acétate (\%), Butyrate (\%)), selon le test de Student-Newman-Keuls.

Acétate (\%) : proportion molaire de l'acétate

Propionate (\%) : Proportion molaire du propionate

Butyrate (\%) : Proportion molaire du butyrate

AGVTot : Acides Gras Volatils totaux

C2/C3 : Ration Acétate/Propionate

Means in the same row with different labels are significantly different for the follow levels of significance : $p<0,01 \%$ (for $A D G$ ) ; $p<0,1 \%$ (for $N-N H 3$ and $p H$ ) according to Student-Newman-Keuls Test.

Tableau 2b : Evolution de la production d'acides gras volatils dans le rumen pendant la période préhivernale et hivernale.

Pattern of volatil fatty acids production in the rumen during early and wet seasons.

\begin{tabular}{lcccc}
\hline & \multicolumn{3}{c}{ Mois } \\
\cline { 2 - 5 } & Mai & Juin & Juillet & Août/Sept. \\
\hline Acétate (mmol/l) & $12,71 \mathrm{~b}$ & $17,84 \mathrm{a}$ & $19,76 \mathrm{a}$ & $19,34 \mathrm{a}$ \\
Acétate (\%) & $76,29 \mathrm{ab}$ & $73,58 \mathrm{ab}$ & $73,22 \mathrm{ab}$ & $72,81 \mathrm{~b}$ \\
Propionate (mmol/l) & $2,32 \mathrm{bcd}$ & $3,44 \mathrm{ab}$ & $3,77 \mathrm{a}$ & $2,66 \mathrm{abcd}$ \\
Propionate (\%) & $13,53 \mathrm{ab}$ & $14,18 \mathrm{ab}$ & $13,72 \mathrm{ab}$ & $9,82 \mathrm{bc}$ \\
Butyrate (mmol/l) & $1,80 \mathrm{bcd}$ & $2,97 \mathrm{abc}$ & $3,56 \mathrm{ab}$ & $4,66 \mathrm{a}$ \\
Butyrate (\%) & $10,20 \mathrm{abc}$ & $12,24 \mathrm{abc}$ & $13,05 \mathrm{abc}$ & $17,37 \mathrm{a}$ \\
AGVTot & $16,83 \mathrm{bc}$ & $24,25 \mathrm{ab}$ & $27,10 \mathrm{a}$ & $26,66 \mathrm{a}$ \\
C2/C3 & $6,20 \mathrm{~b}$ & $5,30 \mathrm{~b}$ & $6,00 \mathrm{~b}$ & $7,60 \mathrm{ab}$ \\
\hline
\end{tabular}

Les valeurs figurant sur la même ligne et ne portant aucun indice commun sont significativement différentes pour : $\mathrm{P}<0,1 \%$ (pour Acétate, Propionate, Propionate (\%), Butyrate, AGVTot, C2/C3); $\mathrm{P}<1 \%$ (pour Acétate (\%), Butyrate (\%)), selon le test de Student-Newman-Keuls.

Acétate (\%) : proportion molaire de l'acétate

Propionate (\%) : Proportion molaire du propionate

Butyrate (\%) : Proportion molaire du butyrate

AGVTot : Acides Gras Volatils totaux

C2/C3 : Ration Acétate/Propionate

Means in the same row with different labels are significantly different for the follow levels of significance : $p<0,01 \%$ (for $A D G$ ) ; $p<0,1 \%$ (for $N-N H 3$ and $p H$ ) according to Student-Newman-Keuls Test. 


\section{EVOLUTION DES PERFORMANCES PONDERALES EN RELATION AVEC LA PRODUCTION DE N-NH ${ }_{3}$ ET DES ACIDES GRAS VOLATILS}

L'évolution pondérale des animaux (figure 2) montre des fluctuations saisonnières avec une croissance ralentie, voire négative en saison sèche chaude et une augmentation spectaculaire du poids vif pendant les périodes de juin-août et de novembre-janvier. Ainsi, des variations saisonnières hautement significatives $(p<0,1 \%)$ ont été observées pour les gains moyens quotidiens (GMQ) avec les plus fortes valeurs enregistrées pendant la saison de pluies et pendant la période post- récolte $(43,2 \mathrm{~g} ; 56,6 \mathrm{~g}$ et $47,6 \mathrm{~g}$, respectivement en juillet-août, novembredécembre et janvier-février).

Les résultats obtenus (tableau 3 , figure 2 ) montrent un lien entre les gains de poids vif, la proportion molaire de propionate et le ratio $\mathrm{C} 2 / \mathrm{C} 3$. Les plus forts gains de poids vif ont été généralement observés pendant les périodes où la proportion molaire de propionate est la plus élevée (mai-juillet et novembre-décembre) et où le rapport C2/C3 est le plus bas. Des corrélations hautement significatives (tableau 3 ) existent entre les GMQ, les proportions molaires de propionate $(r=0,55 ; p<0,1 \%)$ et le ratio C2/C3 $(r=-0,51 ; p<1 \%)$.

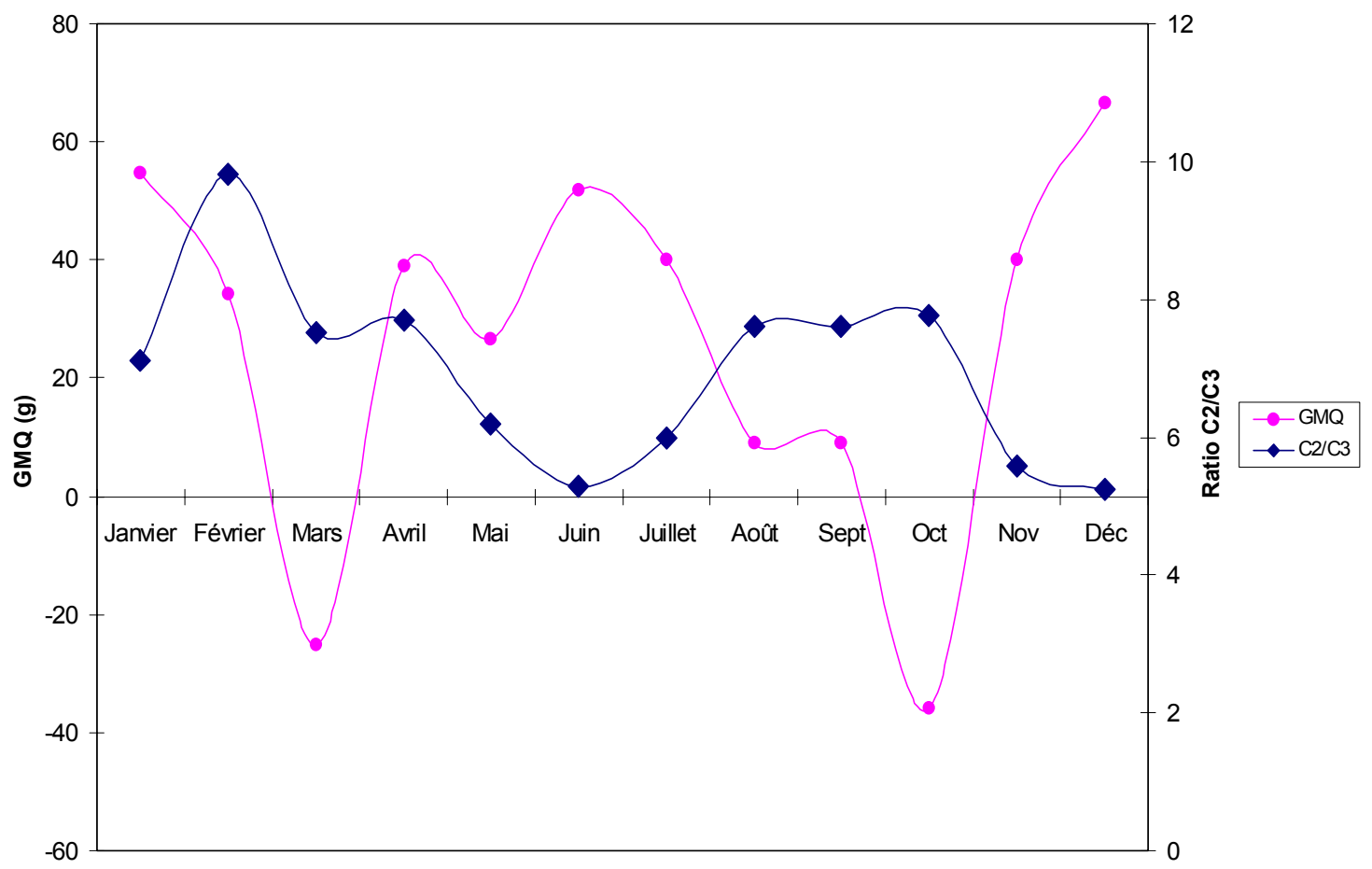

Figure 2 : Evolution des gains moyens quotidiens (GMQ) en relation avec le ratio acétate/propionate C2/C3 : Acétate/propionate ratio

Average Daily Gain (ADG) pattern in relation to Acetate/propionate ratio C2/C3 : Ratio Acetate/propionate 
Tableau 3 : Corrélations entre gain pondéral et paramètres fermentaires.

Correlations between live weight gain and fermentation parameters.

\begin{tabular}{|c|c|c|c|c|c|c|}
\hline & GMQ & N-NH3 & Acétate $(\%)$ & Propionate $(\%)$ & $\mathrm{C} 2 / \mathrm{C} 3$ & $\mathrm{pH}$ \\
\hline GMQ & - & NS & $\begin{array}{c}-0,66 \\
(0,0001)\end{array}$ & $\begin{array}{c}0,55 \\
(0,0006)\end{array}$ & $\begin{array}{c}-0,51 \\
(0,0013)\end{array}$ & NS \\
\hline $\mathrm{N}-\mathrm{NH}_{3}$ & NS & - & $\begin{array}{c}-0,55 \\
(0,0005)\end{array}$ & $\begin{array}{c}0,44 \\
(0,0077)\end{array}$ & $\begin{array}{c}-0,58 \\
(0,0002)\end{array}$ & NS \\
\hline Acétate (\%) & $\begin{array}{c}-0,66 \\
(0,0001)\end{array}$ & $\begin{array}{c}-0,55 \\
(0,0005)\end{array}$ & (2 & $\begin{array}{c}-0,65 \\
(0,0001)\end{array}$ & $\begin{array}{c}0,71 \\
(0,0001)\end{array}$ & NS \\
\hline Propionate $(\%)$ & $\begin{array}{c}0,55 \\
(0,0006)\end{array}$ & $\begin{array}{c}0,44 \\
(0,0077)\end{array}$ & $\begin{array}{c}-0,65 \\
(0,0001)\end{array}$ & - & $\begin{array}{c}-0,98 \\
(0,0001)\end{array}$ & $\begin{array}{c}-0,64 \\
(0,0001)\end{array}$ \\
\hline $\mathrm{C} 2 / \mathrm{C} 3$ & $\begin{array}{c}-0,51 \\
(0,0013)\end{array}$ & $\begin{array}{c}-0,58 \\
(0,0002)\end{array}$ & $\begin{array}{c}0,71 \\
(0,0001)\end{array}$ & $\begin{array}{c}-0,98 \\
(0,0001)\end{array}$ & , & $\begin{array}{c}0,64 \\
(0,0001)\end{array}$ \\
\hline $\mathrm{pH}$ & NS & NS & NS & $\begin{array}{c}-0,64 \\
(0,0001)\end{array}$ & $\begin{array}{c}0,64 \\
(0,0001)\end{array}$ & - \\
\hline
\end{tabular}

GMQ : Gain Moyen Quotidien

Acétate (\%) : Proportion molaire de l'acétate

Propionate (\%): Proportion molaire du propionate

C2/C3 : Ration Acétate/Propionate

\section{DISCUSSION}

\section{ANALYSE DES NIVEAUX DE pH ET $\mathrm{DEN}-\mathrm{NH}_{3}$}

L'augmentation des niveaux de $\mathrm{N}-\mathrm{NH}_{3}$ avec l'arrivée de la saison de pluies est similaire aux observations faites par Sawadogo et al., (1995). Celle-ci serait essentiellement due à la disponibilité en fourrages verts hautement fermentescibles et de qualité relativement bonne en saison de pluies. Cette relation entre la disponibilité de l'azote fermentescible et les niveaux de $\mathrm{N}-\mathrm{NH}_{3}$ a été largement mise en évidence à travers l'utilisation d'urée ou d'autres aliments azotés fermentescibles (Preston, 1986 ; Ali et al., 1997, Nitas et al., 1998). Toutefois, les valeurs moyennes enregistrées en saison de pluies sont inférieures à celles enregistrées (192,50 mg/l) par Sawadogo et al., (1995).

Quant au deuxième pic observé en novembredécembre, il serait essentiellement dû à la bonne disponibilité en résidus de cultures, plus particulièrement ceux liés à la récolte des légumineuses telles le niébé, l'arachide, le voandzou.

Les tendances évolutives observées au niveau du $\mathrm{pH}$, notamment avec les plus fortes valeurs en saison sèche sont en concordance avec celles faites par Sawadogo et al., (1995) avec des animaux de la même race utilisant des parcours naturels.

\section{ANALYSE DE LA PRODUCTION D'ACIDES GRAS VOLATILS}

Les fortes productions d'AGV observées en saison de pluies traduiraient l'effet positif de la relative disponibilité en aliments de qualité, et sont comparables aux observations faites par de nombreux auteurs sur les ovins (Wanapat et al., 1982 ; Hatfield et al., 1998 ; Ouedraogo, 1998) de même que sur les bovins (Stritzler et al., 1998 ; Das et Singh, 1999 ; Delagarde et al., 1999 ; Sahoo et al., 1999 ; Olson et al., 1999 ; Thakur et Sharma, 1999). Les valeurs obtenues sont voisines de celles enregistrées ( $37-46 \mathrm{mmol} / \mathrm{l})$ par Fondevila et al., (1999) sur des ovins, mais elles sont largement inférieures à celles obtenues (50 à plus de $100 \mathrm{mmol} / \mathrm{l}$ ) avec d'autres races et sous diverses conditions alimentaires (Horton et al., 1980 ; Males et Gaskins, 1982 ; Ouedraogo, 1998). Ces faibles valeurs en AGV totaux s'expliqueraient d'une part, par le facteur race, et d'autre part, par les conditions alimentaires relativement pauvres dans notre étude. Ranilla et al., (1997) travaillant sur des ovins a en effet mis en évidence le facteur race, qui traduirait l'aptitude de certaines races à favoriser une plus forte absorption d'AGV à travers les parois du rumen.

L'augmentation significative des proportions de propionate en mai-juillet et en novembredécembre serait imputable à l'abondance relative d'aliments de bonne qualité en ces périodes de l'année. En effet, la période maijuillet correspond à celle où les graminées et les légumineuses annuelles enregistrent leur 
taux maximum en nutriments au niveau de leurs organes foliaires (Penning De Vries et Djiteye, 1982), tandis que la période novembre-décembre correspond à celle où il y a une grande disponibilité en résidus de cultures.

Les valeurs des rapports acétate/propionate enregistrées sont voisines de celles $(4,2$ à $4,8)$ de Wanapat et al., (1982), mais restent supérieures à celles $(0,90$ à 4,18$)$ d'autres auteurs (Horton et al., 1980 ; Ouedraogo, 1998 ; et al., 1999 ; Carro et al., 2000) travaillant sur d'autres races avec des rations incorporant des concentrés azotés et énergétiques. Toutefois, les variations saisonnières du ratio $\mathrm{C} 2 / \mathrm{C} 3$ semblent aller de pair avec celles de la disponibilité en aliments de qualité. Cela est en concordance avec les résultats d'autres travaux (Ouedraogo, 1998 ; Andersen et al., 1999 ; Fondevila et al., 1999) et traduirait le fait que la disponibilité en aliment de bonne valeur nutritive oriente la fermentation microbienne vers des proportions plus accrues de propionate au détriment de l'acétate.

\section{PERFORMANCES PONDERALES EN RELATION AVEC LA PRODUCTION DE $\mathrm{N}-\mathrm{NH}_{3}$ ET DES ACIDES GRAS VOLATILS (AGV)}

L'allure de l'évolution pondérale est en concordance avec celle observée par d'autres auteurs avec des ruminants évoluant sur des parcours naturels (Bicaba et al., 1986 ; Zoundi et al., 1994). Toutefois, les valeurs de GMQ enregistrées sur les parcours naturels restent tout de même inférieures à celles obtenues (70-90 g) sur des animaux de même race soumis à des rations d'embouche (Nianogo et al.,1995). Elles sont toutefois comparables aux GMQ d'animaux de même race soumis à une gestion semi-intensive en pâturage naturel plus une complémentation à 25-50\% des besoins en matière sèche (Nianogo et al., 1997).

Les corrélations significatives observées entre le $G M Q$, le ratio $C 2 / C 3$ et les proportions molaires de propionate sont en concordance avec les observations faites par Salajan et al., (1996). D'une manière générale, les forts gains de poids correspondent également à des périodes où le $\mathrm{N}-\mathrm{NH}_{3}$ est relativement élevé. Cela traduirait l'efficience de l'utilisation de l'énergie métabolisable pour les gains (Reid et al., 1980). Panjanapruthipong et Leng (1998) estiment, que cette situation serait associée à une augmentation de la population bactérienne et de leur efficience dans la digestion.

Les performances pondérales enregistrées pendant la période de septembre-octobre sont les plus faibles de l'année (-22,3 g de GMQ). Ces résultats sont similaires à ceux obtenus par Zoundi et al., (1994) sur des ovins de même race soumis à la croissance compensatrice et indiqueraient une situation réelle de déficit nutritionnel important. Cette période pré-récolte correspond effectivement à un moment où la plupart des espèces annuelles des pâturages de plateau sont en fructification avec un stade de lignification avancé (Penning De Vries et Djiteye, 1982), alors que les parcours des zones inondées ne sont pas encore fréquentables pour les animaux. Cette situation provoquerait une limitation des quantités ingérées, ce qui engendre d'importantes déficiences en terme d'apports énergétiques et azotés. Cela expliquerait la faible production d'acides gras volatils totaux observée pendant le mois d'octobre.

De même, les résultats montrent que les ovins s'adaptent mieux à la période jugée de forte sous-nutrition (mars-juin), comparativement aux bovins pour lesquels de très fortes pertes (parfois $50 \%$ du poids vif) sont enregistrées (Bicaba et al., 1986).

\section{CONCLUSION}

Les parcours naturels, en dépit de leur valeur nutritive jugée faible en général, présentent des situations favorables, particulièrement pendant les périodes de juin-août et de novembre-janvier, où les plus forts taux d'AGV, et les meilleures performances pondérales sont enregistrés.

La situation vécue pendant la période pré-récolte (septembre-octobre) apparaît, néanmoins, préoccupante compte tenu de la faiblesse des produits de la digestion et des pertes de poids vif subies. Cette poche de sous-nutrition raccourcit la période de récupération naturelle (croissance compensatrice) des animaux. Les stratégies d'alimentation des animaux utilisant les parcours naturels devraient privilégier 
l'atténuation de cette poche de sous-nutrition grâce à des apports complémentaires énergétiques et azotées, particulièrement au profit des femelles gestantes dont le pic important de mise-bas est situé entre octobre et décembre. Ces mêmes efforts de complémentation sont requis dans des situations où la croissance compensatrice est utilisée comme stratégie de production de viande ovine en saison de pluies.

La période post-récolte avec l'abondance en résidus de culture, apparaît comme le moment le plus propice de l'année qui pourrait être exploitée dans un système d'alimentation basé sur la complémentation stratégique d'animaux à l'engrais sur parcours naturels. Des apports complémentaires en énergie et en azote protéique permettraient de maximiser les gains dans de tels systèmes de production de viande.

Pendant la saison sèche chaude (mars-mai), les animaux ont accès aux fourrages ligneux riches en azote; les stratégies de complémentation devraient viser la satisfaction de certains besoins liés à la maintenance, la gestation, la production de lait, etc, en mettant l'accent sur des apports équilibrés en énergie et azote fermentescible.

\section{REFERENCES}

ALI (I.), (J. P.) FONTENOT et (V. G.) ALLEN. 1997. Digestibility and nitrogen utilization by sheep fed corn stover treated with different nitrogen sources. Sarhad J. of Agric. 13 (1) : 11-22.

ANDERSEN (J. B.), (J.) SEHESTED and (K. L.) INGVARTSEN. 1999. Effect of dry cow feeding strategy on rumen $\mathrm{pH}$, concentration of volatile fatty acids and rumen epithelieum development. Acta Agriculturae Scandinavica. Section A, Anim. Sci. 49 (3) : 149-155.

BICABA (Z. M.), (R.) BOSMA, (G.) MACON, (M.) SABA et (S. J.) ZOUNDI. 1986. Etude de la croissance compensatrice chez les zébus au Burkina : Analyse comparative après divers degrés de sous-nutrition. Rev. Elev. Méd. pays trop., 39 (3-4) : 414-420.

BOUDET (M.). 1985. Pastures and livestock in arid zone lands. Scientific Reviews on Arid Zone Research 3, 200-207.

CARRO (M. D.), (C.) VALDES, (M. J.) RANILLA and (S. J.) GONZALEZ. 2000. Effect of forage to concentrate ratio in the diet on ruminal fermentation and digesta flow kinetics in sheep offered food at a fixed and restricted level of intake. Anim. Sci. 70 : 127-134.

DELAGARDE (R.), (J. L.) PEYRAUD and (L.) DELABY. 1999. Influence of carbohydrate or protein supplementation on intake, behaviour, and digestion in dairy cows stripgrazing low-nitrogen fertilized perennial ryegrass. Ann. Zootech. 48 (2) : 81-96.

FONDEVILA (M.), (G.) CUFRE, (J. C. M.) NORGUEIRA, (L.) GODIO and (G.) ALCANTU. 1999. Digestion and microbial fermentation of Eragrostis curvula supplemented with tallow. Anim. Sci. 69 (2) : 447-455.

GRYSEELS (G.).1988. Role of livestock on mixed smallholder farms in the Ethiopian Highlands : A case study from Baso and Worena wereda near Debre Berhan. Dissertation, Agricultural university, WAGENINGEN, The Netherlands, 249 p.

GUERIN (H.), (D.) FRIOT, (N. D.) MBAYE, (D.) RICHARD et (A.) DIENG. 1988. Régime alimentaire de ruminants domestiques (bovins, ovins, caprins) exploitant des parcours naturels sahéliens. II. Essai de description du régime par l'étude du comportement alimentaire. Facteurs de variation des choix alimentaires et conséquences nutritionnelles. Revue Elev. Méd. Pays Trop. 41 (4) : 427-440.

HATFIELD (P. G.), (J. A.) HOPKINS, (W. S.) RAMSEY and (A.) GILMORE. 1998. Effects of level of protein and type of molasses on digesta kinetics and blood metabolites in sheep. Small Ruminant Res. 28 (2) : 161-170.

KETELAARS (J. J. H.) and TOLKAMP (B. J.). 1991. Toward a new theory of feed intake regulation in ruminants. Doctoral thesis. Wageningen Agricultural University (The Netherlands), $253 \mathrm{p}$.

KOMISARCZUK (B.) and DURAND (M.). 1991. Effects of mineral on microbial metabolism. In (J.P.) JOUANY (Ed.) Rumen microbial metabolism and ruminant digestion, INRA (France), 179-198.

NIANOGO (A. J.), SOMA (L.), (G. F. X.) BONKOUNGOU, (S.) NASSA et (S. J.) ZOUNDI. 1995. Utilisation optimale de la graine de coton et des fourrages locaux pour l'engraissement des ovins Djallonké type Mossi. Rev. Rés. Amélior. Prod. Agr. Milieu Aride 7 : 179-195.

NIANOGO (A. J.), (S.) NASSA, (L.) SOMA et (H. O.) SANON. 1997. Influence de la complémentation et du mode de conduite sur la croissance des agneaux Mossi en saison pluvieuse. Bull. Anim. Hith. Prod. Afri. 45 : 241-249. 
NITAS (D.), (A.) KARALAZOS and (D.) KOUFIDIS. 1998. Effect of whole cottonseed inclusion in sheep's diet on rumen fermentation. Epitheorese Zootehnikes Epistemes 25 : 45-56.

OLSON (K. C.), (R. C.) COCHRAN, (T. J.) JONES, (T. E. S.) VANZAN, (E. G.) TITGEMEYER and (D.E.) JOHNSON. 1999. Effects of ruminal administration of supplemental degradable intake protein and starch on utilization of low-quality warm-season grass hay by beef steers. J. Anim. Sci. 77 (4) : 1016-1025.

OUEDRAOGO (T.) 1998. Contribution à l'étude de l'utilisation digestive comparée des régimes à base de fourrages pauvres chez le mouton et l'âne. Thèse de Doctorat de $3^{3}$ cycle. Université de Ouagadougou (Burkina Faso), $141 \mathrm{p}$.

ORSKOV (E. R.). 1997. Feed evaluation with emphasis on fibrous roughages and fluctuating suply of nutrients : A Review. Small Ruminant Res. 28 : 1-8.

PENNING DE VRIES (F .W. T.) et DJITEYE (M. A.). 1982. La productivité des pâturages sahéliens : une étude des sols, des végétations et de l'exploitation de cette ressources naturelle. Ed. PUDOC, Wageningen (The Netherlands), $525 \mathrm{p}$.

PRESTON (T. R.). 1986. Better utilization of crop residues and by-products in animal feeding : Research guidelines : 2 - A practical manual for research workers, FAO - Rome (Italie), $154 \mathrm{p}$.

PRITCHARD (R. H.) and (J. R.) MALES. 1982. Effects of supplementation of wheat straw diets twice a day on rumen ammonia, volatile fatty acids and cow performance. J. Anim. Sci. 54 : 1243-1250.

RANILLA (M. J.), (M. D.) CARRO, (C.) VALDES, (F. J.) GIRALDEZ and (S.) LOPEZ. 1997. A comparative study on ruminal activity in Churra and Merino sheep offered alfalfa hay. Anim. Sci. 65 : 121-128.

REID (J. T.), (O. D.) WHITE, (R.) ANRIQUE and (A.) FORTIN. 1980. Nutritional energetics of livestock : some present boundaries of knowledge and future research needs. J .I of Anim. Sci. 51 (6) : 1393-1415.

SALAJAN (G. H.), (D.) MIERLITA and (U.) STANESCU. 1996. The influence of the food structure administrated to fattening lambs in finishing phase on the proportion of volatile fatty acids (VFA) from ruminal juice. Buletinul Universitatii de Stiinte Agricole Cluj Napoca. Seria Zooteh. si Med. Vet. 50 : 37-41.

SAS. 1990. User's guide. SAS Inst . Inc., Cary, N.C., USA, 893-993

SAVADOGO (M.). 2000. Crop residue management in relation to sustainable land use. A case study in Burkina Faso. Dissertation thesis, PhD. Wageningen Agricultural University, The Netherlands, $159 \mathrm{p}$.

SAWADOGO (L. L.), (S. J.) ZOUNDI et (A. J.) NIANOGO. 1995. Analyse de quelques caractéristiques du milieu ruminal d'ovins alimentés sur parcours naturels : incidence d'une complémentation azotée sur les niveaux de $\mathrm{N}-\mathrm{NH}_{3}$ et de $\mathrm{pH}$. Agron. Afr. VII (1) : 34-41.

STRITZLER (N. P.), (J.) WOLSTRUP, (B. O.) EGGUM and (B. B.) JENSEN. 1998. Factors affecting degradation of barley straw in sacco and microbial activity in the rumen of cows fed fibre-rich diets. II. The level of supplemental fishmeal. Anim. Feed Sci. and Technol. 70 : 11-22.

TOUTAIN (B.) 1987. Potentialités pastorales du Nord Burkina Faso. In (A.) GASTON (Ed.). Les pâturages sahéliens de l'Afrique de l'Ouest. Extraits des atlas Elevage et potentialités pastorales sahéliennes. CIRAD-EMVT (France).

Van HOUTERT (M. F. J.). 1993. The production and metabolism of volatile fatty acids by ruminants fed roughages: A Review. Anim. Feed Sci. and Technol. 43 : 189-225.

WANAPAT (M.), (D. O.) ERICKSON and (W. D.) SLANGER. 1982. Nitrogen metabolism in sheep fed protein sources of various solubilities with low quality roughages. J. Anim. Sci. 54 (3) : 625-631.

ZOUNDI (S. J.), (L. L.) SAWADOGO et (A. J.) NIANOGO. 1994. Croissance compensatrice d'ovins alimentés sur parcours naturels : analyse des grains de poids vif et des caractéristiques de la carcasse. Rev. Rés. Amélior. prod. Agr. Milieu Aride 6 : 179-196.

ZOUNDI (S. J.), (A. J.) NIANOGO et (L.) SAWADOGO. 1996. Utilisation de gousses de Piliostigma Reticulatum (DC.) Hochst. et de feuilles de Cajanus cajan (L.) Millsp. en combinaison avec l'urée pour l'engraissement de moutons Djallonké type Mossi et du Sud au Burkina - Tropicultura 14 (4) : 149-152. 